\title{
IDEMPOTENT ELEMENTS OF WEAK PROJECTION GENERALIZED HYPERSUBSTITUTIONS
}

\author{
Nareupanat LEkKoksung and PRAKit Jampachon \\ Department of Mathematics \\ Faculty of Science, Khon Kaen University \\ 40000 Khon Kaen, Thailand \\ e-mail: n.lekkoksung@kkumail.com \\ prajam@kku.ac.th
}

\begin{abstract}
A generalized hypersubstitution of type $\tau=\left(n_{i}\right)_{i \in I}$ is a mapping $\sigma$ which maps every operation symbol $f_{i}$ to the term $\sigma\left(f_{i}\right)$ and may not preserve arity. It is the main tool to study strong hyperidentities that are used to classify varieties into collections called strong hypervarieties. Each generalized hypersubstitution can be extended to a mapping $\hat{\sigma}$ on the set of all terms of type $\tau$. A binary operation on $\operatorname{Hyp}_{\mathrm{G}}(\tau)$, the set of all generalized hypersubstitutions of type $\tau$, can be defined by using this extension. The set $\operatorname{Hyp}_{\mathrm{G}}(\tau)$ together with such a binary operation forms a monoid, where a hypersubstitution $\sigma_{\text {id }}$, which maps $f_{i}$ to $f_{i}\left(x_{1}, \ldots, x_{n_{i}}\right)$ for every $i \in I$, is the neutral element of this monoid. A weak projection generalized hypersubstitution of type $\tau$ is a generalized hypersubstitution of type $\tau$ which maps at least one of the operation symbols to a variable. In semigroup theory, the various types of its elements are widely considered. In this paper, we present the characterizations of idempotent weak projection generalized hypersubstitutions of type $(m, n)$ and give some sufficient conditions for a weak projection generalized hypersubstitution of type $(m, n)$ to be regular, where $m, n \geq 1$.
\end{abstract}

Keywords: idempotent, regular, generalized hypersubstitution, weak projection generalized hypersubstitution.

2010 Mathematics Subject Classification: 08A99, 20M10, 20 M99.

\section{REFERENCES}

[1] A.H. Clifford and G.B. Preston, The Algebraic Theory of Semigroups, Vol. I, no. 2 (The American Mathematical Society, 1964). 
[2] K. Denecke and L. Freiberg, The algebra of strongly full terms, Novi Sad J. Math. 34 (2004) 87-98.

[3] K. Denecke, D. Lau, R. Pöschel and D. Schweigert, Hyperidentities, hyperequational clases and clone congruences, Contribution to General Algebra 7 (Verlag HölderPichler-Tempsky, Wien, 1991) 97-118.

[4] D. Denecke and S. Leeratanavalee, Tree transformations defined by generalized hypersubstitutions, Sci. Math. Jpn. Online 6 (2002) 355-366.

[5] K. Denecke and S.L. Wismath, Hyperidentities and Clones (Gordon and Breach science Publishers, 2000).

[6] K. Denecke and S.L. Wismath, Universal Algebra and Applications in Theoretical Computer Science (Boca Raton: Chapman \& Hall/CRC, 2002).

[7] J. Koppitz and K. Denecke, $M$-Solid Varieties of Algebras (Springer Science+Business Media, Inc., 2006).

[8] S. Leeratanavalee, Structural properties of generalized hypersubstitutions, Kyungpook Math. J. 44 (2004) 261-267.

[9] S. Leeratanavalee, Submonoids of generalized hypersubstitutions, Demonstr. Math. 15 (2007) 13-22.

doi:10.1515/dema-2007-0103

[10] S. Leeratanavalee, Idempotent element of $W_{G}(2,2) \cup\left\{\sigma_{i d}\right\}$, Novi Sad J. Math. 41 (2011) 99-109.

[11] S. Leeratanavalee and K. Denecke, Generalized hypersubstitutions and strongly solid varieties, General Algebra and Applications, Proceeding of the "59th workshop on general algebra 15th conference for young algebraists Potsdam 2000" (Shaker Verlag, 2000) 135-145.

[12] N. Lekkoksung and P. Jampachon, Idempotent elements of pre-generalized hypersubstitutions of type $(m, n)$, Ital. J. Pure Appl. Math. 36 (2016) 827-842.

[13] W. Puninagool and S. Leeratanavalee, Idempotent pre-generalized hypersubstitutions of type $\tau=(2,2)$, An. Ştiinţ. Univ. "Ovidius" Constanţa Ser. Mat. 15 (2007) 55-70.

[14] W. Puninagool and S. Leeratanavalee, The order of generalized hypersubstitutions of type $\tau=(2)$, Int. J. Math. Math. Sci. 2008 (2008) Article ID 263541, 8 pages. doi:10.1155/2008/263541

[15] W. Puninagool and S. Leeratanavalee, The monoid of generalized hypersubstituions of type $\tau=(n)$, Discuss. Math. Gen. Algebra Appl. 30 (2010) 173-191. doi:10.7151/dmgaa.1168

[16] W. Puninagool and S. Leeratanavalee, All regular elements in $H_{y p}(2)$, Kyungpook Math. J. 51 (2011) 139-143. doi:10.5666/KMJ.2011.51.2.139 